\title{
Temperature tolerance and survival in darkness of kelp gametophytes (Laminariales, Phaeophyta): ecological and biogeographical implications
}

\author{
Inka tom Dieck (Bartsch) \\ Biologische Anstalt Helgoland, Notkestr. 31, D-22607 Hamburg, Germany
}

\begin{abstract}
Filamentous red-light grown gametophytes of 47 species of the Laminariales, i.e. about $50 \%$ of all species of the order, were tested for upper survival temperature (UST; 2 wk exposure), and 42 of these species were tested for lower survival temperature (LST; $8 \mathrm{wk}$ exposure). The lowest UST in the order is 19 to $20^{\circ} \mathrm{C}$, encountered in the Arctic to cold-temperate species Laminaria solidungula, Alaria esculenta and Agarum cribrosum. The warm temperate Japanese species Eisenia bicyclis and Undaria pinnatifida have the highest UST of 28 to $30^{\circ} \mathrm{C}$. The UST range in the order Laminariales, thus, spans about $10^{\circ} \mathrm{C}$. This is about $5^{\circ} \mathrm{C}$ less than in the related order Desmarestiales which spans a range in UST of $15^{\circ} \mathrm{C}$. The largest within-family vanation in UST is found in the Alariaceae and Laminariaceae, the 2 families with most species and widest distribution. The Alariaceae span a range in UST of $10^{\circ} \mathrm{C}$, the Laminariaceae of 7 to $8^{\circ} \mathrm{C}$, the Lessoniaceae of 5 to $6^{\circ} \mathrm{C}$, the Phyllariaceae of $4^{\circ} \mathrm{C}$ and the Chordaceae of 3 to $4^{\circ} \mathrm{C}$. All gametophytes tested survive a temperature of $1^{\circ} \mathrm{C}$ for $8 \mathrm{wk}$. Eleven out of 42 species do not tolerate -1.5 and/or $0^{\circ} \mathrm{C}$ over $8 \mathrm{wk}$. Temperature tolerance of the gametophytes coincides with the large-scale distribution of the species, but is not crucial for distribution limits in most cases. In addition to the temperature tolerance, the dark tolerance of filamentous gametophytes of 12 kelp species was investigated. All but 3 species survived 18 mo of darkness.
\end{abstract}

\section{INTRODUCTION}

In recent years temperature tolerance and temperature responses of growth and reproduction have been experimentally determined in many marine macroalgal species (e.g. Lüning 1984, Cambridge et al. 1987 , Guiry et al. 1987, Lüning \& Freshwater 1988, Wiencke \& tom Dieck 1989, 1990, Orfanidis 1991, Peters \& Breeman 1992,1993). The main purpose of these investigations was to answer questions in ecological and historical biogeography, as well as a general interest in autecology (e.g. van den Hoek 1982, Breeman 1988, van den Hoek \& Breeman 1990).

During these investigations, thermal ecotypes have mostly been detected in species with a tropical to temperate distribution area but seldom in species with a temperate distribution area. Generally, temperature tolerance did not vary to the same extent as temperature effects on growth and reproduction (review: Breeman 1988). It was hypothesized that temperature tolerance is a rather conservative character (Lüning \& Freshwater 1988). As such, it is utilized in phylogenetic considerations.

Despite the large numbers of temperature tolerance studies the knowledge of the variation range of this parameter within algal orders is scarce. The purpose of this study was to test the upper survival temperature of as many species of the Laminariales as possible including those which grow in the most extreme temperature environments known for this order. Furthermore, it was to evaluate whether this character is as variable as the biogeographical distribution range from Arctic to warm temperate waters and the great morphological diversity of the order suggest.

\section{MATERIAL AND METHODS}

Temperature tolerance. The investigated Laminariales species are listed in Table 1. Temperature 
Table 1. List of Laminariales gametophytes used in temperature tolerance and darkness survival tests. Locations: Aus = Victoria, Australia; Bam1., Bam2 = Bamfield, Canada; Bra = Espirito Santo, Brazil $;$ Bri = Roscoff, Brittany, France; Cal = California, USA; Cal1 = Pillar Point, California, USA; Cal2 = Santa Barbara, California, USA; Chi1= Mehuin, Valdivia, Chile; Chi2 = Mar Brava, Chiloé, Chile; Far = Faeroe Islands; Hok = Hokkaido, Japan; Hax = Halifax, Canada; Hel = Helgoland, North Sea, Germany; Icl = Tjörnes, Iceland; Iglo = Igloolik, Canada; IOM = Isle of Man; Irl = Ireland; Irl1 = Branock Isl., Ireland; Jap = Japan; NewZ = New Zealand; Nfl = Newfoundland, Canada; SAfr1 = Oudekraal, Cape Peninsula, South Africa; SAfr2 = Lamberts Bay, South Africa; Tasm1 = Tinderbox Bay, Tasmania, Australia; Tasm2 = Safety Cove, Tasmania, Australia. Culture numbers = male and female gametophyte respectively

\begin{tabular}{|c|c|c|c|}
\hline Species & & Location & Culture number \\
\hline \multicolumn{4}{|c|}{ Làminariaceae, genus Laminaria } \\
\hline L. abyssalis & Joly \& Oliveira Filho & Bra & $1291 / 1292$ \\
\hline L. angustata & Kjellman & Hok & $1035 / 1036$ \\
\hline \multirow{2}{*}{ L. bongardiana } & Postels \& Ruprecht & $\begin{array}{l}\text { Jap } \\
\text { Bam1 }\end{array}$ & $1204 / 1205$ \\
\hline & & Bam2 & $1109 / 1110$ \\
\hline L. coriacea & Miyabe & Jap & $1223 / 1224$ \\
\hline L. diabolica & Miyabe & Jap & $1225 / 1226$ \\
\hline L. digitata & (Hudson) Lamouroux & Hel & $1003 / 1004$ \\
\hline L. ephemera & Setchell & Bam & 1120 \\
\hline L. farlowii & Setchell & Cal2 & $1053 / 1054$ \\
\hline L. hyperborea & (Gunnerus) Foslie & Hel & $1001 / 1002$ \\
\hline L. japonica & Areschoug & Hok & $1029 / 1030$ \\
\hline L. Iongicruris & Bach. Pylaie & Far & $1145 / 1146$ \\
\hline L. longissima & Miyabe & Hok & $1037 / 1038$ \\
\hline L. ochroleuca & De la Pylaie & Bri & $1262 / 1263$ \\
\hline L. pallida & (Greville) J. Agardh & SAfr 1 & $1266 / 1267$ \\
\hline L. religiosa & Miyabe & Jap & $1231 / 1232$ \\
\hline \multirow[t]{2}{*}{ L. saccharina } & (Linnaeus) Lamouroux & Hel & $1005 / 1006$ \\
\hline & & $\mathrm{IOM}$ & $1007 / 1008$ \\
\hline L. schinzii & Foslie & SAfr2 & $1264 / 1265$ \\
\hline L. setchellii & Silva & Bam & $1212 / 1213$ \\
\hline L. sinclairii & $\begin{array}{l}\text { (Harvey ex Hooker f. \& Harvey) } \\
\text { Farlow, Anderson \& Eaton }\end{array}$ & Call & $1047 / 1048$ \\
\hline L. solidungula & J. Agardh & Iglo & $1099 / 1100$ \\
\hline \multicolumn{4}{|c|}{ Laminariaceae (except genus Laminaria) } \\
\hline Agarum cribrosum & (Mertens) Bory & Iglo & $1105 / 1106$ \\
\hline Costaria costata & (Turner) Saunders & Jap & $1239 / 1240$ \\
\hline Cymathere triplicata & (Postels \& Ruprecht) J. Agardh & Bam & $1125 / 1126$ \\
\hline Kjellmaniella crassifolia & Miyabe & Jap & $1241 / 1242$ \\
\hline K. gyrata & (Kjellman) Miyabe & Jap & $1255 / 1256$ \\
\hline Pleurophycus gardneri & Setchell \& Saunders & Bam & $1223 / 1124$ \\
\hline \multicolumn{4}{|l|}{ Alariaceae } \\
\hline Alaria crassifolia & Kjellman & Jap & $1251 / 1252$ \\
\hline A. esculenta & (Linnaeus) Greville & $\mathrm{Icl}$ & $1019 / 1020$ \\
\hline A. marginata & Postels \& Ruprecht & Call & $1049 / 1050$ \\
\hline \multirow[t]{2}{*}{ Ecklonia radiata } & (C. Agardh) J. Agardh & Aus & $1193 / 1194$ \\
\hline & & Tasm 1 & $1253 / 1254$ \\
\hline Eisenia arborea & Areschoug & Bam & $1208 / 1209$ \\
\hline E. bicyclis & (Kjellman) Setchell & Jap & $1237 / 1238$ \\
\hline Pterygophora californica & Ruprecht & Bam & $1129 / 1130$ \\
\hline Undaria pinnatifida & (Harvey) Suringar & Hok & $1041 / 1042$ \\
\hline \multicolumn{4}{|l|}{ Lessoniaceae } \\
\hline Dictyoneuropsis reticulata & (Saunders) G. M. Smith & Cal & $1163 / 1164$ \\
\hline Lessonia corrugata & Lucas & $\operatorname{Tasm} 2$ & $1257 / 1258$ \\
\hline L. nigrescens & Bory & Chil & $1245 / 1246$ \\
\hline L. trabeculata & Villouta \& Santelices & Chi2 & $1247 / 1248$ \\
\hline Macrocystis angustifolia & Bory & Aus & $1259 / 1260$ \\
\hline \multirow[t]{2}{*}{ M. integrifolia } & Bory & Bam & $1135 / 1136$ \\
\hline & & Cal & $1155 / 1156$ \\
\hline M. pyrifera & (Linneaus) C. Agardh & $\begin{array}{l}\text { Cal } \\
\text { Newz }\end{array}$ & $\begin{array}{l}1159 / 1160 \\
1182 / 1183\end{array}$ \\
\hline \multirow{2}{*}{$\begin{array}{l}\text { Nereocystis luetkeana } \\
\text { Pelagophycus porra }\end{array}$} & (Mertens) Postels \& Ruprecht & $\mathrm{Cal}$ & $1206 / 1207$ \\
\hline & (Leman) Setchell & $\mathrm{Cal}$ & $1161 / 1162$ \\
\hline \multicolumn{4}{|l|}{ Chordaceae } \\
\hline Chorda filum & (Linnaeus) Stackhouse & Irl & $1195 / 1196$ \\
\hline C. tomentosa & Lyngbye & $\mathrm{Hel}$ & 1261 \\
\hline \multicolumn{4}{|l|}{ Phyllariaceae } \\
\hline Saccorhiza dermatodea & (Bach. Pylaie) J. Agardh & Nfl & 1190 \\
\hline S. polyschides & (Lightfoot) Batters & Irl1 & $1191 / 1192$ \\
\hline \multicolumn{4}{|c|}{${ }^{a}$ L. groenlandia sensu Druehl (1968) } \\
\hline
\end{tabular}


tolerance of filamentous red-light grown gametophytes was determined in glass tubes $155 \mathrm{~mm}$ high, $25 \mathrm{~mm}$ diameter) with plastic, nontransparent lids filled with $20 \mathrm{ml}$ von-Stosch-enriched autoclaved seawater (VSES: modified after Guiry \& Cunningham 1984, iodide enrichment after Tatewaki 1966). The glass tubes were immersed in temperature-controlled Haake D8-V water baths (variation $\pm 0.1^{\circ} \mathrm{C}$ ) for $2 \mathrm{wk}$ (upper temperature tolerance) or $8 \mathrm{wk}$ (lower temperature tolerance). The upper temperature tolerance was determined in steps of $1^{\circ} \mathrm{C}$ and the tests were carried out 2 to 4 times. The lower temperature tolerance was tested at $-1.5,0$ and $1{ }^{\circ} \mathrm{C}$ and the tests were usually repeated once. The gametophytes were inoculated in the test tubes without prior acclimatization to elevated or reduced temperatures. The gametophyte stock cultures were maintained at $10^{\circ} \mathrm{C}$, except for Laminaria solidungula, Saccorhiza dermatodea, Alaria esculenta and Agarum cribrosum which were maintained at $1^{\circ} \mathrm{C}$. A photon fluence rate of $30 \mu \mathrm{mol} \mathrm{m} \mathrm{m}^{-2} \mathrm{~s}^{-1}$ at the surface of the water baths was provided by fluorescent white light (Osram L 20W/19, Daylight de Luxe) for $12 \mathrm{~h} \mathrm{~d}^{-1}$. The cultures received fresh prewarmed medium twice a week during the test period. The survival limits were determined after a postculture of $4 \mathrm{wk}$ in Provasoli's enriched seawater (PES; Provasoli 1968) at $10^{\circ} \mathrm{C}$ and $16 \mathrm{~h}$ light $\mathrm{d}^{-1}$ at photon fluence rates lower than $20 \mu \mathrm{mol} \mathrm{m} \mathrm{m}^{-2} \mathrm{~s}^{-1}$. The survival temperature was defined as that temperature at which living cells could be detected under the microscope after postcultivation. Part of the experiments was also conducted by using an automatic test system described by Lüning et al. (1987).

Dark tolerance. Dark tolerance of filamentous redlight grown gametophytes of 12 Laminariales species was qualitatively determined over a period of $18 \mathrm{mo}$. Similar amounts of thalli of female and male gametophytes of each species were inoculated in glass tubes (55 $\mathrm{mm}$ high, $25 \mathrm{~mm}$ diameter) with nontransparent plastic lids, filled with $20 \mathrm{ml}$ PES and placed in darkness. The temperature was 0 or $8( \pm 1)^{\circ} \mathrm{C}$. Each month 1 tube of each species was transferred to white fluorescent light $\left(16 \mathrm{~h}\right.$ light $\left.\mathrm{d}^{-1}\right)$, while the remaining tubes were provided with fresh medium and then put back into darkness. Thus, the dark cultures were subjected to light once a month for about $10 \mathrm{~min}$.

Survival was established after 1 or 2 mo in postcultivation conditions (Table 4). If the gametophytes were still vegetative after 1 mo they were further cultivated in most cases. Gametophytes were examined under the microscope. Resumed growth of gametophytes or formation of sporophytes was considered as recovery.

\section{RESULTS}

\section{Temperature tolerance}

The upper and lower temperature tolerance of filamentous gametophytes of 47 species of Laminariales after 2 wk exposure to the experimental temperatures is shown in Table 2. There was a difference in upper survival temperature (UST) of $10^{\circ} \mathrm{C}$ within all tested species of the order Laminariales. The lowest UST was $20^{\circ} \mathrm{C}$ (Laminaria solidungula, Agarum cribrosum, Alaria esculenta) while the highest UST was $30^{\circ} \mathrm{C}$ (Eisenia bicyclis from Japan). Of the Laminariaceae 26 species were tested, comprising about $50 \%$ of the species of this family. They exhibited a range in UST of $7{ }^{\circ} \mathrm{C}$, from 20 (L. solidungula) to $26-27^{\circ} \mathrm{C}$ (L. japonica, Costaria costata). Within the Alariaceae 8 species were tested, comprising about $25 \%$ of the species of this family. Their range in UST varied between 20 ( $\mathrm{A}$. esculenta) and $30^{\circ} \mathrm{C}$ (E. bicyclis), i.e. a $10^{\circ} \mathrm{C}$ difference as in the whole order Laminariales. The 2 species of the Chordaceae survived 24 to 25 (Chorda tomentosa) and 27 to $28^{\circ} \mathrm{C}$ (C. filum), i.e. a 3 to $4{ }^{\circ} \mathrm{C}$ difference in the family. Within the Lessoniaceae 9 species were tested, which is about $55 \%$ of the species of the family. Their UST ranged from 22 to 23 (Dictyoneuropsis reticulata) to $26^{\circ} \mathrm{C}$ (Macrocystis pyrifera), a $4^{\circ} \mathrm{C}$ difference in upper temperature tolerance for this family. Two out of the 4 species of the Phyllariaceae were tested. Their UST were 21 (Saccorhiza dermatodea) and $24^{\circ} \mathrm{C}$ (S. polyschides).

Usually there was a difference in UST of 0 or $1^{\circ} \mathrm{C}$ between repetitive experiments of the same gametophyte stock culture (Table 2 ). In some cases this deviation was, however, $2^{\circ} \mathrm{C}$. Differences in UST of male and female gametophytes of the same species were observed, but no general trend emerged. In 10 species the male gametophyte had a higher temperature tolerance than the female, in 7 cases it was vice versa and in all other tests male and female gametophytes exhibited the same UST.

The lower survival temperature (LST) of 42 tested Laminariales gametophytes varied only between -1.5 and $1{ }^{\circ} \mathrm{C}$ under the specified conditions (Table 2). Male and female gametophytes of 25 species survived $-1.5^{\circ} \mathrm{C}$ over $8 \mathrm{wk}$, while gametophytes of 11 species only survived 0 to $1{ }^{\circ} \mathrm{C}$ and died at $-1.5^{\circ} \mathrm{C}$. Male and female gametophytes of 6 species showed different survival limits.

The change in UST of Laminaria gametophytes as a function of incubation time is shown for 2 species in Table 3 . There was a 3 to $5^{\circ} \mathrm{C}$ difference in UST between $1 \mathrm{~d}$ and $8 \mathrm{wk}$ exposure experiments. The most rapid decline in temperature tolerance was between $1 \mathrm{~d}$ and $1 \mathrm{wk}$ experimental time. There was little 
Table 2. Temperature tolerance of Laminariales gametophytes. Upper temperature tolerance (UST) and lower temperature tolerance (LST) were determined after $4 \mathrm{wk}$ of postcultivation in $10^{\circ} \mathrm{C}$ after 2 or $8 \mathrm{wk}$ exposure to the experimental temperatures, respectively. TR: no. of repeated treatments for UST Underlining at UST indicates the most frequent survival temperature Locations as in Table 1

\begin{tabular}{|c|c|c|c|c|c|c|}
\hline \multirow[t]{2}{*}{ Species } & \multirow{2}{*}{ Location } & \multicolumn{2}{|c|}{ Male } & \multicolumn{2}{|c|}{ Female } & \multirow[t]{2}{*}{ TR } \\
\hline & & LST & UST & LST & UST & \\
\hline \multicolumn{7}{|c|}{ Laminariaceae, genus Laminaria } \\
\hline L. abyssalis & Bra & & & & $24-\underline{26}^{2}$ & 3 \\
\hline \multirow{2}{*}{ L. angustata } & & $0-1$ & 25 & 1 & 25 & 1 \\
\hline & $\begin{array}{l}\text { Hok } \\
\text { Jap }\end{array}$ & -1.5 & $23-\underline{25}$ & $\begin{array}{l}-1.5 \\
-1.5\end{array}$ & $\begin{array}{l}24-\underline{26} \\
25-26\end{array}$ & $\begin{array}{l}3 \\
2\end{array}$ \\
\hline \multirow[t]{2}{*}{ L. bongardiana } & Bam 1 & & $20-22$ & & $19-\underline{21}$ & 3 \\
\hline & Bam2 & -1.5 & $20-\underline{21}$ & 0 & 20 & 3 \\
\hline L. coriacea & Jap & -1.5 & 25 & -1.5 & $24-25$ & 2 \\
\hline L. diabolica & Jap & -1.5 & $25-26$ & & & 2 \\
\hline L. digitata & $\begin{array}{l}\text { Hel } \\
\text { Hax }\end{array}$ & -1.5 & $\begin{array}{c}23 \\
23-24\end{array}$ & -1.5 & $\begin{array}{c}23 \\
22-23\end{array}$ & $\begin{array}{l}3 \\
4\end{array}$ \\
\hline \multirow{3}{*}{$\begin{array}{l}\text { L. ephemera } \\
\text { L. farlowii } \\
\text { L. hyperborea }\end{array}$} & Bam & & & $-1.5^{b}$ & $22-23^{b}$ & 2 \\
\hline & Cal2 & -1.5 & $23-24$ & -1.5 & $21-22-23$ & 4 \\
\hline & Hel & -1.5 & $22-\underline{23}$ & & & 3 \\
\hline \multirow{8}{*}{$\begin{array}{l}\text { L. japonica } \\
\text { L. longicruris } \\
\text { L. longissima } \\
\text { L. ochroleuca } \\
\text { L. pallida } \\
\text { L. religiosa } \\
\text { L. saccharina }\end{array}$} & Hok & -1.5 & $26-27$ & -1.5 & $25-26$ & 2 \\
\hline & Far & -1.5 & 24 & -1.5 & 24 & 3 \\
\hline & Hok & & $\underline{24}-25$ & & $24-\underline{25}$ & 3 \\
\hline & $\mathrm{Bri}$ & 1 & 25 & $0-1$ & 25 & 3 \\
\hline & SAfr1 & -1.5 & $23-\underline{25}$ & 1 & 25 & 3 \\
\hline & Jap & -1.5 & $24-\overline{26}$ & -1.5 & $25-26$ & 3 \\
\hline & Hel & -1.5 & $23-24$ & -1.5 & $23-25$ & 2 \\
\hline & IOM & & $23-24$ & & $23-\underline{24}$ & 3 \\
\hline \multirow{4}{*}{$\begin{array}{l}\text { L. schinzii } \\
\text { L. setchellii } \\
\text { L. sinclainii } \\
\text { L. solidungula }\end{array}$} & SAfr2 & $-1.5-1$ & $24-\underline{25}$ & $-1.5-1$ & 25 & 3 \\
\hline & Bam & -1.5 & 23 & -1.5 & 23 & 3 \\
\hline & Cal1 & -1.5 & $22-\underline{23}$ & -1.5 & 23 & 3 \\
\hline & Iglo & -1.5 & 20 & -1.5 & $19-20$ & 2 \\
\hline \multicolumn{7}{|c|}{ Laminariaceae (except genus Laminaria) } \\
\hline \multirow{5}{*}{$\begin{array}{l}\text { Agarum cribrosum } \\
\text { Costaria costata } \\
\text { Cymathere triplicata } \\
\text { Kjellmaniella crassifolia } \\
\text { K. gyrata }\end{array}$} & Iglo & & $\underline{20}-21$ & & & 3 \\
\hline & Jap & 0 & $25-\underline{27}$ & -1.5 & $\underline{2} 6-27$ & 3 \\
\hline & Bam & -1.5 & 22 & & & 3 \\
\hline & Jap & -1.5 & $\underline{25}-26$ & -1.5 & 26 & 3 \\
\hline & Jap & -1.5 & $\underline{25}-26$ & 0 & $25-26$ & 4 \\
\hline Pleurophycus gardneri & Bam & 0 & 22 & 0 & 22 & 2 \\
\hline \multicolumn{7}{|l|}{ Alariaceae } \\
\hline Alaria crassifolia & Jap & -1.5 & 26 & -1.5 & $24-26$ & 3 \\
\hline A. esculenta & $\mathrm{Icl}$ & -1.5 & $19-\underline{20}$ & -1.5 & $\underline{20}-21$ & 3 \\
\hline A. marginata & Call & -1.5 & $\underline{21-22}$ & -1.5 & $\underline{22}-23$ & 3 \\
\hline \multirow{2}{*}{ Ecklonia radiata } & Aus & $1->1$ & 28 & $1->1$ & 27 & 2 \\
\hline & Tasm1 & & 28 & & $27-28$ & 2 \\
\hline Eisenia arborea & Bam & $0-1$ & $25-27$ & $1->1$ & $25-27$ & 3 \\
\hline E. bicyclis & Jap & 1 & $29-30$ & 1 & 29 & 2 \\
\hline Pterygophora californica & Bam & -1.5 & 25 & -1.5 & 24 & 2 \\
\hline Undaria pinnatifida & Hok & $<0$ & $28-29$ & $<0$ & $27-\underline{28}-29$ & 4 \\
\hline Lessoniaceae & & & & & & \\
\hline Dictyoneuropsis reticulata & a Cal & & 23 & & $22-23$ & 2 \\
\hline Lessonia corrugata & $\operatorname{Tasm} 2$ & & 23 & & 23 & 2 \\
\hline L. nigrescens & Chit & & 24 & & $23-24$ & 2 \\
\hline L. trabeculata & Chi2 & $0-1$ & $23-25$ & $>1$ & $23-\underline{25}$ & 3 \\
\hline Macrocystis angustifolia & Aus & 0 & 25 & $0-1$ & $23-\overline{24}$ & 2 \\
\hline M. integrifolia & $\begin{array}{l}\text { Bam } \\
\text { Cal }\end{array}$ & $\begin{array}{l}-1.5 \\
-1.5\end{array}$ & $\begin{array}{c}24-25 \\
24\end{array}$ & 1 & 24 & $\begin{array}{l}2 \\
2\end{array}$ \\
\hline M. pyrifera & Cal & 1 & $25-26$ & $0-1$ & 25 & 2 \\
\hline & NewZ & & & & 24 & 2 \\
\hline Nereocystis luetkeana & $\mathrm{Cal}$ & -1.5 & 23 & -1.5 & 23 & 2 \\
\hline Pelagophycus porra & Cal & $0-1$ & $24-25$ & $0-1$ & 24 & 2 \\
\hline Chordaceae & & & & & & \\
\hline Chorda filum & Irl & -1.5 & $26-28$ & -1.5 & $\underline{27}-28$ & 3 \\
\hline C. tomentosa & Hel & & & $-1.5^{b}$ & $24-25^{b}$ & 2 \\
\hline Phyllariaceae & & & & & & \\
\hline Saccorhiza dermatodea & $\mathrm{Nfl}$ & & & $-1.5^{b}$ & $21^{b}$ & 2 \\
\hline S. polyschides & Irl1 & $1->1$ & $23-24$ & $0-1$ & $23-\underline{24}$ & 3 \\
\hline
\end{tabular}


Table 3. Laminaria digitata and L. schinzii. Upper survival temperature of Laminaria gametophytes exposed $1 \mathrm{~d}$ to $8 \mathrm{wk}$ to the experimental temperatures. Results were obtained after 4 wk of postcultivation in $10^{\circ} \mathrm{C}$. $x$ : all gametophytes survived; $\cdot$ : gametophytes partly damaged, but regenerated; -: gametophytes dead, ?: no data

\begin{tabular}{|c|c|c|c|c|c|c|c|c|c|c|c|}
\hline \multirow[t]{2}{*}{ Species } & \multirow[t]{2}{*}{ Sex } & \multirow{2}{*}{$\begin{array}{c}\text { Exposure } \\
\text { time }\end{array}$} & \multicolumn{9}{|c|}{ Experimental temperatures $\left({ }^{\circ} \mathrm{C}\right)$} \\
\hline & & & 21 & 22 & 23 & 24 & 25 & 26 & 27 & 28 & 29 \\
\hline \multirow[t]{14}{*}{ L. digitata } & $M$ & $1 \mathrm{~d}$ & & & & $x$ & $x$ & $x$ & $x$ & $x$ & - \\
\hline & & $2 \mathrm{~d}$ & & & & $x$ & $x$ & $x$ & - & - & - \\
\hline & & $4 \mathrm{~d}$ & & & $x$ & $x$ & . & - & - & & \\
\hline & & $1 \mathrm{wk}$ & & $x$ & $\hat{x}$ & $\hat{x}$ & - & - & - & & \\
\hline & & $2 w k$ & & $x$ & $\hat{x}$ & . & - & - & & & \\
\hline & & $4 \mathrm{wk}$ & & $x$ & $x$ & - & - & - & & & \\
\hline & & $8 \mathrm{wk}$ & $x$ & $x$ & . & - & - & & & & \\
\hline & $\mathrm{F}$ & $1 d$ & & & & $x$ & $x$ & $x$ & . & - & - \\
\hline & & $2 \mathrm{~d}$ & & & & $\hat{x}$ & $x$ & . & - & - & - \\
\hline & & $4 \mathrm{~d}$ & & & $x$ & $\hat{x}$ & . & - & - & & \\
\hline & & $1 \mathrm{wk}$ & & $x$ & $x$ & . & - & - & - & & \\
\hline & & $2 w k$ & & $x$ & $x$ & $?$ & - & - & & & \\
\hline & & $4 \mathrm{wk}$ & & $x$ & . & - & - & - & & & \\
\hline & & $8 w k$ & $x$ & - & . & - & - & & & & \\
\hline \multirow[t]{14}{*}{ L. schinzii } & $M$ & $1 \mathrm{~d}$ & & & & & $x$ & $x$ & $x$ & - & - \\
\hline & & $2 d$ & & & & $x$ & $x$ & $x$ & - & - & - \\
\hline & & $4 d$ & & & & $x$ & $x$ & - & - & - & \\
\hline & & 1 wk & & & $x$ & $x$ & . & - & - & - & \\
\hline & & $2 w k$ & & & $x$ & $x$ & . & - & - & - & \\
\hline & & $4 w k$ & & $x$ & $x$ & . & . & - & - & & \\
\hline & & $8 w k$ & $\times$ & $x$ & $x$ & . & - & - & & & \\
\hline & F & $1 d$ & & & & & $x$ & $x$ & . & . & - \\
\hline & & $2 d$ & & & & $x$ & $x$ & $x$ & . & . & - \\
\hline & & $4 \mathrm{~d}$ & & & & $x$ & $x$ & - & - & - & \\
\hline & & 1 wk & & & $x$ & $x$ & $x$ & - & - & - & \\
\hline & & $2 w k$ & & & $x$ & $x$ & . & - & - & - & \\
\hline & & $4 w \mathrm{k}$ & & $x$ & $x$ & . & - & - & - & & \\
\hline & & $8 w k$ & $x$ & $x$ & . & - & - & - & & & \\
\hline
\end{tabular}

8, indicating that the limit of long-term survival temperature was not yet reached after $8 \mathrm{wk}$ for Laminaria gametophytes.

\section{Dark tolerance}

The development of filamentous gametophytes of 12 species of Laminariales after 6 to $18 \mathrm{mo}$ in darkness is shown in Table 4. All survived a period of 16 mo in darkness while most species even survived 18 mo. Only Laminaria bongardiana, L. hyperborea and $L$. saccharina did not regenerate within 1 mo of postcultivation after 18 mo of darkness. The gametophytes of the 3 species that were cultivated at $0^{\circ} \mathrm{C}$ were in a good condition even after 18 mo of darkness, and the postcultivation confirmed this result. From Month 9 in darkness onwards gametophytes were often bleached when they were transferred to light. However, the state of the gametophytes at the end of the dark treatment was not recorded in more detail and, thus, is not indicated in Table change in survival temperature between 1 and $2 \mathrm{wk}$ but UST decreased by 1 to $2^{\circ} \mathrm{C}$ between Weeks 2 and
4. After 1 or 2 mo postcultivation there was, however, some regeneration in all species up to Month 16, indi-

Table 4. Dark tolerance of filamentous red-light grown Laminariales gametophytes. Development of gametophytes in postcultivation conditions [PCC, $(\mathrm{mo}) ; 0$ or $8^{\circ} \mathrm{C}, 16 \mathrm{~h}$ light $\mathrm{d}^{-1}$ ] after 6 to $18 \mathrm{mo}$ of dark treatment. TID: time in darkness (mo); $\mathrm{v}$ : gametophytes in PCC vegetative but alive; S: gametophytes formed sporophytes in PCC, - most of inoculum bleached; -: experiment was terminated after 1 mo in PCC, no further observation

\begin{tabular}{|c|c|c|c|c|c|c|c|c|c|c|c|c|c|c|c|c|c|c|}
\hline \multirow[t]{2}{*}{ Temp. $\left({ }^{\circ} \mathrm{C}\right)$} & \multirow[t]{2}{*}{ Species } & \multicolumn{2}{|c|}{6} & \multicolumn{2}{|c|}{7} & \multicolumn{2}{|c|}{8} & \multicolumn{2}{|c|}{9} & \multicolumn{2}{|c|}{10} & \multicolumn{2}{|c|}{12} & \multicolumn{2}{|c|}{15} & \multicolumn{2}{|c|}{16} & \multirow{2}{*}{$\begin{array}{c}18 \\
1\end{array}$} \\
\hline & & 1 & 2 & 1 & 2 & 1 & 2 & 1 & 2 & 1 & 2 & 1 & 2 & 1 & 2 & 1 & 2 & \\
\hline \multirow[t]{3}{*}{0} & Alaria esculenta & $\mathrm{v}$ & - & $\mathrm{V}$ & - & $\mathrm{v}$ & - & $\mathrm{v}$ & - & $\mathrm{v}$ & - & $\mathrm{v}$ & - & $\mathrm{v}$ & - & $\mathrm{v}$ & $\mathrm{v}$ & $\mathrm{v}$ \\
\hline & Laminaria solidungula & $\mathrm{S}$ & - & $\mathrm{S}$ & - & $\mathrm{S}$ & - & $\mathrm{S}$ & - & $\mathrm{s}$ & - & $\mathrm{S}$ & - & $\mathrm{S}$ & - & $\mathrm{v}$ & $\mathrm{S}$ & $\mathrm{S}$ \\
\hline & Saccorhiza dermatodea & $\mathrm{v}$ & - & $\mathrm{v}$ & - & $\mathrm{v}$ & - & $\mathrm{v}$ & - & $\mathrm{v}$ & - & $v$ & - & $v$ & - & $\mathrm{v}$ & - & $v$ \\
\hline \multirow[t]{9}{*}{8} & & $S$ & - & $\mathrm{S}$ & - & $\mathrm{s}$ & - & $\mathrm{S}$ & - & $\mathrm{s}$ & - & $\mathrm{s}$ & - & $\mathrm{s}$ & - & S & - & $\mathrm{S}$ \\
\hline & L. bongardiana & $\mathrm{v}$ & $\mathrm{S}$ & $\mathrm{v}$ & - & $\mathrm{v}$ & $\mathrm{S}$ & $\mathrm{S}$ & - & $\mathrm{S}$ & - & - & $\mathrm{S}$ & - & $\mathrm{S}$ & - & $\mathrm{S}$ & - \\
\hline & L. hyperborea & $\mathrm{S}$ & - & $\mathrm{v}$ & $\mathrm{S}$ & $\mathrm{S}$ & - & $\mathrm{S}$ & - & . & $\mathrm{S}$ & $\mathrm{S}$ & - & - & $\mathrm{S}$ & . & $S^{a}$ & - \\
\hline & L. ochroleuca & $\mathrm{S}$ & - & $\mathrm{v}$ & $\mathrm{s}$ & $\mathrm{v}$ & - & $\mathrm{v}$ & - & $\mathrm{v}$ & $\mathrm{S}$ & - & $\mathrm{S}$ & $\mathrm{S}$ & - & $=$ & $\mathrm{S}$ & $\mathrm{v}$ \\
\hline & L. pallida & $\mathrm{S}$ & - & $\mathrm{v}$ & $\mathrm{S}$ & S & - & $\mathrm{S}$ & - & $\mathrm{S}$ & - & $\mathrm{S}$ & - & * & S & - & $\mathrm{S}$ & $\mathrm{v}$ \\
\hline & L. saccharina & $\mathrm{S}$ & - & $\mathrm{S}$ & - & $\mathrm{S}$ & - & $\mathrm{S}$ & - & $\mathrm{S}$ & - & $\mathrm{S}$ & - & $\mathrm{S}$ & - & . & $\mathrm{S}$ & - \\
\hline & L. schinzii & $\mathrm{S}$ & - & $\mathrm{S}$ & - & $\mathrm{S}$ & - & $\mathrm{S}$ & - & $\mathrm{S}$ & - & . & S & $\mathrm{S}$ & - & . & $\mathrm{S}$ & $\mathrm{v}$ \\
\hline & \multirow{2}{*}{$\begin{array}{l}\text { L. setchellii } \\
\text { S. polyschides }\end{array}$} & $\mathrm{S}$ & - & S & - & $\mathrm{S}$ & - & $\mathrm{S}$ & - & $\mathrm{v}$ & $\mathrm{S}$ & $\mathrm{S}$ & - & S & - & $\mathrm{v}$ & $S$ & $\mathrm{v}$ \\
\hline & & $\mathrm{S}$ & - & $\mathrm{S}$ & - & $\mathrm{S}$ & - & S & - & $\mathrm{S}$ & - & $\mathrm{S}$ & - & $\mathrm{S}$ & - & - & $\mathrm{S}$ & $\mathrm{S}$ \\
\hline
\end{tabular}


cated by the development of vegetative gametophyte thalli or sporophytes.

\section{DISCUSSION}

\section{Methodological aspects}

Several methodological aspects have to be taken into account when evaluating the results. Table 3 indicates the influence of the experimental duration for the determination of the upper temperature tolerance. Even after 2 wk the survival limit did not remain constant but was reduced by another 1 to $2^{\circ} \mathrm{C}$ after 8 wk and even then a constant value was not obtained. Other studies comparing 2 wk and 12 wk exposure times in subtropical and cold-temperate species obtained similar results (Cambridge et al. 1987, 1990, Novaczek \& Breeman 1990, Novaczek et al. 1990) while the red alga Polyneura hilliae exhibited a constant upper survival temperature from the second week onwards that did not change over the following $6 \mathrm{wk}$ (Yarish et al. 1987). This indicates that dependency of UST on its incubation period is not the same in different taxonomic groups. For biogeographical considerations a test period of $12 \mathrm{wk}$ will probably better simulate a summer season in the field than of 2 wk. For practical reasons, however, 2 wk exposure times were chosen for this study.

An acclimatization of the inoculated material probably would have resulted in slightly higher UST values than obtained during the present investigation. The gametophytes of 3 Laminariales species which were acclimated for at least $4 \mathrm{wk}$ to $20^{\circ} \mathrm{C}$ prior to the experiment exhibited UST which were 0.5 to $1.5^{\circ} \mathrm{C}$ higher than of the same species tested here (compare Tables 2 \& 5i Peters \& Breeman 1992, 1993). This could, however, also be an effect of the different day lengths used in both studies.

Besides treatment duration and acclimatization, the light period and light intensity may also alter the temperature tolerance. Photon fluence rates of $40 \mu \mathrm{mol} \mathrm{m} \mathrm{m}^{-2}$ $\mathrm{s}^{-1}$ may reduce the UST by several degrees compared to $20 \mu \mathrm{mol} \mathrm{m}^{-2} \mathrm{~s}^{-1}$ (e.g. Yarish et al. 1986, Cambridge et al. 1987, 1990). Furthermore short day length may improve the long-term survival in low temperatures and alter it in both ways in high temperatures (e.g. Cambridge et al. 1984, 1987, Yarish et al. 1984, 1986). During this study intermediate day length $112: 12 \mathrm{~h}$ light:dark cycle) and photon fluence rates $(30 \mu \mathrm{mol}$ $\mathrm{m}^{-2} \mathrm{~s}^{-1}$ ) were chosen. Thus, there is the possibility that UST obtained during this investigation are slightly lower than under long-day (16:8 h light:dark) conditions and LST are slightly higher than under short-day (8:16 h light:dark) conditions.
Filamentous gametophytes which were used during the present investigation were of a spherical form and consisted of very many vegetative cells. Thus, the amount of inoculated material differed from test to test. At the upper 2 survival temperatures the outer cells of the inoculum were regularly damaged or dead whereas the inner part was still alive and regenerated during postcultivation. The initial size of the inoculum perhaps was critical for the survival limit and may partly account for the variation of the results. Bolton \& Lüning (1982) compared the upper temperature tolerance of freshly released zoospores and filamentous gametophytes of Laminaria saccharina. The filamentous gametophytes were damaged at $23^{\circ} \mathrm{C}$ but regenerated from a few cells, while the zoospores died at $23^{\circ} \mathrm{C}$ supporting the idea that filamentous gametophytes yield higher survival values than zoospores. Similar differences became obvious in Saccorhiza polyschides. The lower survival temperature of female gametophytes directly derived from zoospores was $3^{\circ} \mathrm{C}$ after 2 wk (Norton 1977) while the filamentous gametophytes used in the present investigation survived $1^{\circ} \mathrm{C}$ over 8 wk (Table 2).

It is difficult to decide whether temperature ecotypes exist in kelp gametophytes as different isolates were tested only in a few cases and mostly not during the same study. All species that were tested with 2 isolates in this study (Laminaria angustata, L. digitata, L. bongardiana, Ecklonia radiata, Macrocystis integrifolia, M. pyrifera; Table 2) either exhibited no differences between isolates, or exhibited differences that were within the range of experimental error. The same upper survival limits were also found in different studies for different isolates of $L$. saccharina and $L$. longicruris (Bolton \& Lüning 1982), Chorda tomentosa from eastern and western Atlantic (compare Tables $2 \& 5$; Novaczek et al. 1986), $M$. angustifolia from southern Australia and South Africa (compare Tables 2 \& 5; Branch 1974), Lessonia nigrescens and L. trabeculata (compare Tables 2 \& 5i Peters \& Breeman 1993) as well as between northern and southern New Zealand isolates of $E$. radiata (Table 5; Novaczek 1984). However, E. radiata from New Zealand exhibited an upper temperature tolerance of only 24 to $25^{\circ} \mathrm{C}$ (Novaczek 1984) while Tasmanian and southern Australian isolates survived 27 to $28^{\circ} \mathrm{C}$ (Table 2). E. radiata sensu Womersley (1967) is a broad definition for a variable species (review of taxonomical problems: Bolton \& Anderson 1993) and the differing survival limits may indicate that more than one taxon is involved.

Macrocystis pynifera of the southwest Pacific region only inhabits cold temperate regions such as southern Tasmania or southern New Zealand. This implies a lower UST than for $M$. pyrifera isolates from southern California. During this investigation the New Zealand isolate survived $24^{\circ} \mathrm{C}$ and, thereby, exhibited a $1{ }^{\circ} \mathrm{C}$ 
Table 5. Compiled literature data on lower and upper survival temperature (LST/UST) of Laminariales gametophytes. ${ }^{\circ}$ Growth limit Locations: Chi3 = Tierra del Fuego, Chile; Chin = China $;$ Esp = Espegrend, Norway; GB = Great Britain; LIS = Long Island Sound, USA; Med = Mediterranean Sea; Per = San Juan, Peru; SAfr = South Africa; other locations as in Table 1

\begin{tabular}{|c|c|c|c|c|}
\hline Species & Location & $\mathrm{LST} / \mathrm{UST}\left({ }^{\circ} \mathrm{C}\right)$ & $\begin{array}{l}\text { Duration of } \\
\text { experiment }\end{array}$ & Source \\
\hline \multicolumn{5}{|l|}{ Chordaceae } \\
\hline Chorda filum & $\operatorname{Hax}$ & $126-<28$ & ? & Novaczek et al. (1986) \\
\hline C. tomentosa & $\operatorname{Hax}$ & $/ 24-<26$ & $?$ & Novaczek et al. (1986) \\
\hline \multicolumn{5}{|l|}{ Phyllariaceae } \\
\hline Phyllariopsis brevipes & Med & $>5 /<25$ & ? & Henry (1987) \\
\hline Saccorhiza polyschides & GB & $>3 / 25$ & $2 w k$ & Norton (1977) \\
\hline \multicolumn{5}{|l|}{ Laminariaceae } \\
\hline Laminaria abyssalis & Bra & $/ 24-26$ & $2 w k$ & de Oliveira \& de Oliveira (1987) \\
\hline \multirow[t]{3}{*}{ L. digitata } & $\mathrm{Hel}$ & $/ 22-23$ & $2 w k$ & Bolton \& Lüning (1982) \\
\hline & $\operatorname{Hax}$ & $/ 22-23$ & $2 \mathrm{wk}$ & Bolton \& Lüning (1982) \\
\hline & $\mathrm{Hel}$ & 120 & $1 \mathrm{wk}$ & Lüning (1980) \\
\hline \multirow[t]{2}{*}{ L. hyperborea } & Hel & $/ 21$ & $2 \mathrm{wk}$ & Bolton \& Lüning (1982) \\
\hline & $\mathrm{Hel}$ & 120 & $1 \mathrm{wk}$ & Lüning $(1980)$ \\
\hline \multirow[t]{3}{*}{ L. longicruris } & $\operatorname{Hax}$ & $/ 23$ & $2 w \mathrm{k}$ & Bolton \& Lüning (1982) \\
\hline & Iglo & $/ 23$ & $2 w \mathrm{k}$ & Bolton \& Lüning (1982) \\
\hline & LIS & $/ 25$ & $12 \mathrm{~d}$ & Egan et al. (1989) \\
\hline \multirow[t]{2}{*}{ L. japonica } & Chin & $1>24$ & $?$ & Fang et al. (1963) \\
\hline & $?$ & $0 / 25-27$ & & Cheng (1969) \\
\hline L. pallida & SAfr & $/ 24.5-<26.5$ & $30 d$ & Branch (1974) \\
\hline L. religiosa & Jap & 125 & $10 \mathrm{~d}$ & Funano (1983) \\
\hline \multirow[t]{6}{*}{ L. saccharina } & Hel & 123 & $2 w \mathrm{k}$ & Bolton \& Lüning (1982) \\
\hline & IOM & $/ 23$ & $2 \mathrm{wk}$ & Bolton \& Lüning (1982) \\
\hline & Bri & 123 & $2 w k$ & Bolton \& Lüning (1982) \\
\hline & Esp & $/ 23$ & $2 w k$ & Bolton \& Lüning (1982) \\
\hline & Hel & $/ 21$ & $1 \mathrm{wk}$ & Lüning (1980) \\
\hline & $\mathrm{GB}$ & $/ 22$ & $?$ & Kain $(1969)$ \\
\hline L. solidungula & Iglo & $/ 18-20$ & $2 w k$ & Bolton \& Lüning (1982) \\
\hline \multicolumn{5}{|l|}{ Alariaceae } \\
\hline Ecklonia biruncinata & SAfr & $/ 26$ & $7 \mathrm{~d}$ & Bolton \& Anderson (1987) \\
\hline \multirow[t]{2}{*}{ E. maxima } & SAfr & $/ 25-<28$ & $7 \mathrm{~d}$ & Bolton \& Anderson (1987) \\
\hline & SAfr & $/ 24.1-<26.5$ & $30 \mathrm{~d}$ & Branch $\{1974\}$ \\
\hline E. radiata & NewZ & $\cdot 8-9 / 24-25$ & $?$ & Novaczek (1984) \\
\hline Eisenia arborea & Bam & $>3 /$ & ? & Maier (1984) \\
\hline \multirow[t]{3}{*}{ Undaria pinnatifida } & Jap & $-1 / 27.5-<30$ & $?$ & Akiyama (1965) \\
\hline & Jap & 130 & $?$ & Saito (1962) (in Saito 1975) \\
\hline & Med & $/ 29.5-29.7$ & $2 \mathrm{wk}$ & Peters \& Breeman (1992) \\
\hline \multicolumn{5}{|l|}{ Lessoniaceae } \\
\hline \multirow[t]{2}{*}{ Macrocystis pyrifera } & Cal & $>5 /$ & $?$ & Maier (1984) \\
\hline & Chil & $-2 / 24.5$ & $4 / 2 w k$ & Peters \& Breeman (1993) \\
\hline M. angustifolia & SAft & $/ 24.1-26.5$ & $30 \mathrm{~d}$ & Branch $(1974)$ \\
\hline Lessonia vadosa & Chi3 & $-2 / 19.9-21.4$ & $4 / 2 w k$ & Peters \& Breeman (1993) \\
\hline L. nigrescens & Chi1 & $-2 / 24.6$ & $4 / 2 w \mathrm{k}$ & Peters \& Breeman (1993) \\
\hline L. trabeculata & Per & $-2 / 25.7-26.5$ & $4 / 2 w k$ & Peters \& Breeman (1993) \\
\hline
\end{tabular}

lower UST than the Californian isolate which is within the range of variability between experiments and thus, not significant. However, in a detailed study, Hay (1990) demonstrated that $M$. pyrifera does not persist at its geographical limit in New Zealand in areas where maximum summer temperatures exceed 18 to $19^{\circ} \mathrm{C}$ for several days, whereas in southern North America, $M$. pyrifera extends to areas where seasurface maxima reach $27^{\circ} \mathrm{C}$ for several days. Here, a temperature ecotype for the sporophyte has been described (North 1972), which indicates that the game- tophytes of a more southern isolate of $M$. pyrifera may also survive higher temperatures than 25 to $26^{\circ} \mathrm{C}$ as determined during this investigation. This either implies temperature ecotypes in $M$. pyrifera or nonconspecificity of Australian and Californian isolates.

\section{Dark survival}

The ability of 12 kelp species to survive 18 mo in darkness may have been influenced by the fact that 
filamentous gametophytes were used. It was striking that often only the inner cells of a gametophyte inoculum were still pigmented after the prolonged dark treatment and regenerated during postcultivation. Embryo spores of Laminaria saccharina, L. digitata and L. hyperborea did not survive $15 \mathrm{mo}$ in darkness (Lüning 1980)

The gametophytes of Laminaria solidungula, Alaria esculenta and Saccorhiza dermatodea which were kept at $0^{\circ} \mathrm{C}$ during the dark experiment were fully pigmented even after 18 mo of darkness and probably would have survived much longer dark periods, while the gametophytes that were kept at $8^{\circ} \mathrm{C}$ were for the most part bleached after 18 mo and only partly regenerated with few cells. This indicates that low temperatures may favour survival in darkness. As no species was tested at both temperatures in this study, the exact interrelation between temperature and survival in darkness remains to be tested. In $L$. hyperborea, zoospores which were tested at 10 and $17^{\circ} \mathrm{C}$ survived darkness slightly better in the cooler condition (Kain 1964).

It is not known whether filamentous gametophytes exist in nature, thereby forming a 'seed-bank' of microscopic forms. Such as a 'seed-bank' is, however, probable as the extensive dark tolerance of gametophytes (Table 4) and their ability to grow vegetatively under low light conditions will enable survival of those gametophytes that do not become fertile due to insufficient light or temperature conditions. With the ability of filamentous gametophytes to form sporophytes even after prolonged periods of darkness if light conditions become favourable again (Table 4) another requirement for a 'seed-bank' is fulfilled.

\section{Temperature tolerance and biogeographical considerations}

The upper survival temperature of Laminariales gametophytes ranges between $19-20$ and $29-30^{\circ} \mathrm{C}$ (Tables $2 \& 5$ ) as determined during this investigation and including literature data. This $10^{\circ} \mathrm{C}$ range in UST of kelp gametophytes was determined in more than $50 \%$ of the species of the order including species of the most extreme cold and warm temperate waters inhabited by the Laminariales. Thus, one may expect that these data are representative for the whole order and that the survival range would not be substantially altered even if more species were tested.

The gametophytes of the 3 Arctic to cold-temperate northern hemisphere kelp species Laminaria solidungula, Agarum cribrosum and Alaria esculenta (Table 2) show the lowest upper survival limit $\left(19\right.$ to $20^{\circ} \mathrm{C}$ ) of all investigated species. The same low survival limit is only known from Lessonia vadosa which inhabits the southernmost tip of South America (Peters \& Breeman 1992). This is remarkable because the sporophytes of some northeastern Pacific kelp species (e.g. Cymathere triplicata, Pleurophycus gardneri) have been characterized as especially stenothermal with upper survival temperatures of 15 to $17^{\circ} \mathrm{C}$ (Lüning \& Freshwater 1988), but their gametophytes survive $22^{\circ} \mathrm{C}$. A temperature tolerance of $20^{\circ} \mathrm{C}$ is relatively high for $L$. solidunguld, and this is further evidence for the probable conservative character of this parameter. The Arctic endemic species $L$. solidungula will be subject to water temperatures of only -1.5 up to $5^{\circ} \mathrm{C}$ throughout the year. Although it also occurs off Newfoundland, a region with higher sea-surface temperatures, it is restricted here to deep water (Lüning 1990) or to fjords with temperatures near freezing point (Hooper 1984). As it is assumed that the Laminariales evolved in the North Pacific and that some cold-temperate species entered the North Atlantic only after the first inundation of the Bering land bridge about 3.5 million yr ago (Lüning 1990, Lüning \& tom Dieck 1990), adaptation to Arctic conditions in this group could not have started before the beginning of the last glaciation period about 2 million yr ago. This time period probably was not long enough to permit a further decrease in upper temperature tolerance in $L$. solidungula gametophytes. Even gametophytes of Antarctic Desmarestiales, which potentially had 10 million yr more time for cold adaptation because of the earlier formation of icecovered Antarctica, still survive 13 to $16^{\circ} \mathrm{C}$ in similar test conditions although they only encounter temperatures of up to $5^{\circ} \mathrm{C}$ during the course of the year in nature (Wiencke \& tom Dieck 1989).

In the South Atlantic there are no cold stenothermal kelps as in the northern hemisphere, except for Lessonia vadosa extending from Patagonia to Tierra del Fuego (Peters \& Breeman 1992). The gametophytes of the Brazilian species of Laminaria and of the South African species of Laminaria, Ecklonia and Macrocystis had an upper survival limit of 25 to $26^{\circ} \mathrm{C}$ (Tables 2 \& 5) and only partly survived $-1.5^{\circ} \mathrm{C}$. The genus Laminaria probably originated in the northern hemisphere, and the high temperature tolerance of Brazilian and South African Laminaria species (25 to $26^{\circ} \mathrm{C}$ ) is consistent with the hypothesis that amphiequatorial distribution of temperate species with a heteromorphic life history results from long-distance dispersal of vegetative gametophytes (microthalli), as proposed by Peters \& Breeman (1992) for amphiequatorial Desmarestiales gametophytes. According to paleoclimatic reconstructions, tropical summer seasurface temperatures during the last glaciation period were about 24 to $26^{\circ} \mathrm{C}$ in the coldest regions, i.e. the eastern Atlantic and eastern Pacific (CLIMAP Project 
Members 1981) thereby permitting passage of warmtolerant microthalli. This hypothesis is supported by species of all other genera of the Laminariales with amphiequatorial distribution. The gametophytes of the southern hemisphere species all show UST of at least $25^{\circ} \mathrm{C}$ (e.g. Ecklonia radiata: $28^{\circ} \mathrm{C}$, Eisenia arborea: 25 to $27^{\circ} \mathrm{C}$. Macrocystis pyrifera: 24 to $25^{\circ} \mathrm{C}$ ). As vegetative microthalli of the Laminariales are very persistent, may survive prolonged periods of darkness and thereby do not lose their ability to form sporophytes (Table 4), even a recent dispersal via the tropics by cooler deep-water currents may be taken into consideration.

There is a generally high survival limit of Japanese kelp species which reflects the extreme temperature conditions at the Japanese coasts. The Japanese coastline is characterized by compressed isotherms, by strong annual temperature fluctuations and a rapid change of temperature conditions from north to south (Funahashi 1973, Lüning 1990).

The Japanese species with the lowest UST of 24 to $25^{\circ} \mathrm{C}$, Laminaria angustata, L. longissima and $L$. coriacea, are confined to the cooler Pacific side of Hokkaido and northern Honshu while the characteristic kelp species of the warm areas of Central and South Honshu and Kyushu tolerate temperatures of 28 to $30^{\circ} \mathrm{C}$ (Undaria pinnatifida and Eisenia bicyclis; Table 2). E. bicyclis and Ecklonia cava (not investigated) extend to the Pacific side of South Kyushu with an annual variation of temperatures between 17 and $27^{\circ} \mathrm{C}$ (Chihara 1975) and $U$. pinnatifida grows on the slightly cooler west coast of Kyushu (Saito 1972). Outside Japan, a temperature tolerance as high (27 to $28^{\circ} \mathrm{C}$ ) was found only in Ecklonia radiata isolates from southern Australia and Tasmania. This species, which grows in warm temperate regions of Australia and New Zealand, extends to the southernmost coral reefs of western Australia (28 to $29^{\circ} \mathrm{C}$ ) with mean monthly water temperatures of 20 to $25^{\circ} \mathrm{C}$ (Hatcher et al. 1987). Thus, in all species with very high UST, the distribution limit seems to be determined by upper lethal limits of the gametophyte and/or sporophyte. It is not expected that any gametophyte of the Laminariales will survive more than $30^{\circ} \mathrm{C}$ over a prolonged time.

An upper survival limit of $29^{\circ} \mathrm{C}$ seems to be sufficiently high for a transfer via the tropical belt as was outlined by Peters \& Breeman (1992) for Undaria pinnatifida, a species recently introduced to New Zealand and Tasmania. It may thus be only a question of time until other warm-temperate kelp species, such as Eisenia bicyclis, may be accidentally introduced with ballast water for instance to warm temperate coastlines outside Japan.

It is striking that all investigated Japanese isolates of the Laminariales have consistently higher survival limits than related taxa of the northern hemisphere. The same phenomenon was observed in amphi-oceanic taxa of Desmarestia, D. ligulata and $D$. viridis, with Japanese isolates showing higher upper survival limits than other northern hemisphere strains of the same species (Peters \& Breeman 1992). The explanation may be found in glacial conditions. In Japan, large annual temperature ranges were also present to some extent during glaciations, in contrast to the eastern Pacific and eastern and western North Atlantic (van den Hoek \& Breeman 1990). Thus, in Japan selection pressure has probably consistently operated in improving tolerance to high temperatures in contrast to all other regions.

The upper survival temperature of all tested Laminariales gametophytes shows striking discrepancies between temperature tolerance of the gametophytes and the distribution limit of the species. Some species with a high temperature tolerance are not distributed as close to the Equator as other species with a lower or similar temperature tolerance, and vice versa. Northeastern Pacific species clearly show this phenomenon. The 2 cold-temperate species Agarum cribrosum and Cymathere triplicata with an upper survival limit of 21 to $22^{\circ} \mathrm{C}$ do not extend further south than San Juan Island and Whidbey Island respectively, while Laminaria bongardiana with an upper survival limit of $21^{\circ} \mathrm{C}$ is distributed as far south as Oregon and has isolated populations in California (Druehl 1970). Similarly the southern distribution limit of several northern species, such as Macrocystis integrifolia, L. setchellii, Alaria marginata, Nereocystis luetkeana, L. sinclairii and Costaria costata, is situated near Point Conception, but this cannot be explained by their gametophyte upper temperature tolerance which ranges from 21-22 to $26-27^{\circ} \mathrm{C}$. Other species with a similar temperature tolerance of 23 to $25^{\circ} \mathrm{C}$, such as L. farlowil and Pterygophora californica, extend $5^{\circ}$ further south to the Baja California peninsula (Druehl 1970).

The available data for Laminariales indicate that the upper temperature tolerance of sporophytes is 1 to $7^{\circ} \mathrm{C}$ lower than that of gametophytes (compare Tables $2 \& 5$ with: Sundene 1962, Munda \& Lüning 1977, Lüning 1984, Novaczek et al. 1986, Lüning \& Freshwater 1988, tom Dieck 1992, tom Dieck \& de Oliveira 1993). As lethal limits of the non-hardiest stage (often macrothalli) in a life cycle were found to be irrelevant in explaining distribution limits in many seaweeds (Breeman 1988), distribution in Laminariales often is probably determined by temperature requirements for gametogenesis (being relevant for the formation of sporophytes) or reproduction rather than by temperature tolerance of gametophytes or sporophytes. Exceptions may be found either in annual species that survive the unfavourable season with the help of the hardiest stage, the microthallus (e.g. Chorda filum and 
C. tomentosa; Breeman 1988) or in those species whose distribution may be influenced by the El Niño as outlined by Peters \& Breeman (1993) for South American Lessonia trabeculata and $L$. nigrescens, or in species inhabiting very warm waters, such as Undaria pinnatifida, Eisenia bicyclis and Ecklonia radiata (see above).

\section{CONCLUSION}

The range in upper temperature tolerance of only $10^{\circ} \mathrm{C}$ for 1 specific stage of the life cycle of the Laminariales seems to be low for an entire order. The closely related order Desmarestiales spans at least $15^{\circ} \mathrm{C}$ in UST for the gametophytes. Gametophytes of Antarctic Desmarestia anceps, D. menziesii, Phaeurus antarcticus and Himanthothallus grandifolius survived 13,16 to 17,15 to 16 and 15 to $16^{\circ} \mathrm{C}$ respectively (Wiencke \& tom Dieck 1989, 1990) and gametophytes of cold-temperate South African D. firma survived 22 to $<26^{\circ} \mathrm{C}$ (Anderson \& Bolton 1989). Eleven South American and northern hemisphere Desmarestia species were tested with upper survival limits of gametophytes between 21.8 and $28.5^{\circ} \mathrm{C}$ (Peters \& Breeman 1992 , 1993). Considering the distribution of the genus Desmarestia (Anderson \& Bolton 1989, Lüning 1990), it is not expected that this range will increase by much even if more species were tested. Comparison with other algal orders is difficult due to insufficient data. In the red algal order Gigartinales, the genus Gigartina alone comprises species with upper survival limits ranging between 15 and $33^{\circ} \mathrm{C}$, a $18^{\circ} \mathrm{C}$ difference in total (Guiry et al. 1987, Wiencke \& tom Dieck 1990). In this case it is, however, not certain that the same phase in the life cycle was tested for all species (i.e. gametophyte or tetrasporophyte). The primitive brown algal order Ectocarpales could have a still broader range as members of this order are distributed in all oceans. Existing data for different isolates of the species Ectocarpus siliculosus (Bolton 1983) and for Antarctic/cold temperate Geminocarpus geminatus (Wiencke \& tom Dieck 1990) already span a range in UST of $19^{\circ} \mathrm{C}$.

Recent research into the morphology (Mathieson et al. 1981), historical biogeography (Lüning \& tom Dieck 1990), hybridization (Sanbonsuga \& Neushul 1978, Lewis 1991) and molecular biology (Bhattacharya \& Druehl 1988, Fain et al. 1988, Stam et al. 1988, Bhattacharya et al. 1991, Saunders \& Druehl 1991, 1992) of the Laminariales provides increasing evidence for a recent radiation of kelps. This was outlined explicitly by Saunders \& Druehl (1992) who assume a radiation time of 16 to 20 million yr ago or at most 30 million yr ago. The small range in temperature tolerance of Laminariales gametophytes may be a further indication for the young phylogenetic age of the order.
Acknowledgements. I thank $K$. Lüning for supplying the Laminariales gametophytes of the culture collection that could only established with the additional help of the following colleagues: J. J Bolton, L. Druehl, T Funano, I. Maier, H. Nakahara, M. Neushul, D. G. Müller, E. C. de Oliveira, Y Sanbonsuga, M. Tatewaki, J. Waaland. Special thanks to P. Kadel who supported me with her help during the temperature tolerance tests. I thank A. F. Peters and K. Lüning for valuable suggestions on an earlier draft of the manuscript and $M$. Dring for his critical review.

\section{LITERATURE CITED}

Akiyama, K. (1965). Studies of ecology and culture of Undaria pinnatifida (Harv.) Sur. II. Environmental factors affecting the growth and maturation of gametophyte. Bull. Tohoku reg. Fish. Res. Lab. 25: 143-170

Anderson, R. J., Bolton, J. J. (1989). Growth and fertility, in relation to temperature and photoperiod, in South African Desmarestia firma (Phaeophyceae). Botanica mar. 32: $149-158$

Bhattacharya, D., Druehl, L. D. (1988). Phylogenetic comparison of the small-subunit ribosomal DNA sequence of Costaria costata (Phaeophyta) with those of other algae, vascular plants and Oomycetes. J. Phycol. 24: 539-543

Bhattacharya, D., Mayes, C. F., Druehl, L. D. (1991). Restriction endonuclease analysis of ribosomal DNA sequence variation in Laminaria (Phaeophyta). J. Phycol. 27: 624-628

Bolton, J. J. (1983). Ecoclinal variation in Ectocarpus siliculosus (Phaeophyceae) with respect to temperature growth optima and survival limits. Mar. Biol. 73: 131-138

Bolton, J. J., Anderson, R. J. (1987). Temperature tolerance of two southern African Ecklonia species (Alariaceae: Laminariales) and of hybrids between them. Mar. Biol. 96: 292-297

Bolton, J. J., Anderson, R. J. (1993). Ecklonia. In: Akatsuka, I. (ed.) Biology of economic seaweeds. In press

Bolton, J. J., Lüning, K. (1982). Optimal growth and survival temperatures of Atlantic Laminaria species (Phaeophyta) in culture. Mar. Biol. 66: 89-94

Branch, M. L. (1974). Limiting factors for the gametophytes of three South African Laminariales. Sea Fisheries Branch Investigational Report 104. Galvin \& Sales, Cape Town, p. $1-38$

Breeman, A. M. (1988). Relative importance of temperature and other factors in determining geographic boundaries of seaweeds: experimental and phenological evidence. Helgoländer Meeresunters. 42: 199-241

Cambridge, M., Breeman, A. M., Kraak, S., van den Hoek, C (1987). Temperature responses of some tropical to warm temperate Cladophora species in relation to their distribution in the North Atlantic Ocean. Helgoländer Meeresunters. 41: 329-354

Cambridge, M., Breeman, A. M., van den Hoek, C. (1984). Temperature responses of some North Atlantic Cladophora species (Chlorophyceae) in relation to their geographical distribution. Helgoländer Meeresunters. 38: $349-363$

Cambridge, M., Breeman, A. M., van den Hoek, C. (1990). Temperature responses limiting the geographical distribution of two temperate species of Cladophora (Cladophorales: Chlorophyta) in the North Atlantic ocean. Phycologia 29: 74-85

Cheng, T (1969). Production of kelp - a major aspect of China's exploitation of the sea. Econ. Bot. 23: 215-236

Chihara, M. (1975). Geographic distribution of marine algae 
in Japan. In: Tokida, J., Hirose, H. (eds.) Advance of phycology in Japan. VEB Gustav Fischer Verlag, Jena, p. $241-253$

CLIMAP Project Members (1981). Seasonal reconstructions of the earth's surface at the last glacical maximum. The geological Society of America Map and Chart Series MC-36. Washington, DC

de Oliveira, E. C., de Oliveira, M. C. (1987). Tolerancia de gametofitos de Laminaria abyssalis (Phaeophyta) a differentes temperaturas, in vitro. Neritica, Pontal do Sul, PR, 28 (supl.): 153-158

Druehl, L. D. (1968). Taxonomy and distribution of northeast Pacific species of Laminaria. Can. J. Bot. 21: 199-207

Druehl, L. D. (1970). The pattern of Laminariales distribution in the Northeast Pacific. Phycologia 9: 237-247

Egan, B., Vlasto, A., Yarish, C. (1989). Seasonal acclimation to temperature in Laminaria longicruris de la Pyl. (Phaeophyta). J. exp. mar. Biol. Ecol. 129: 1-16

Fain, S. R., Druehl, L. D., Baillie, D. L. (1988). Repeat and single copy sequences are differentially conserved in the evolution of kelp chloroplast DNA. J. Phycol. 24: 292-302

Fang, T. C., Wu, C., Jiang, B. Y., Li, J. J., Ren, K. Z. (1963). The breeding of a new variety of haidai (Laminaria japonica Aresch.). Scientia sin. 12: 1011-1018

Funahashi, S. (1973). Distribution of marine algae in the Japan sea, with special reference to the phytogeographical positions of Vladivostok and Noto peninsula districts. J. Fac. Sci. Hokkaido Univ. (Ser. 5) 10: 1-31

Funano, T. (1983). The ecology of Laminaria religiosa Miyabe. I. The life history and the alternation of nuclear phases of Laminaria religiosa, and the physiological ecology of the gametophytes and the embryonal sporophytes. Scient. Rep. Hokkaido Fish. exp. Stn 25: 61-109

Guiry, M. C., Cunningham, E. M. (1984). Photoperiodic and temperature responses in the reproduction of northeastern Atlantic Gigartina acicularis (Rhodophyta: Gigartinales). Phycologia 23: 357-367

Guiry, M. D., Tripodi, G., Lüning, K. (1987). Biosystematics, genetics and upper temperature tolerance of Gigartina teedii (Rhodophyta) from the Atlantic and Mediterranean. Helgoländer Meeresunters. 41: 283-295

Hatcher, B. G., Kirkman, H., Wood, W. F. (1987). Growth of the kelp Ecklonia radiata near the northern limit of its range in western Australia. Mar. Biol. 95: 63-73

Hay, C. H. (1990). The distribution of Macrocystis (Phaeophyta: Laminariales) as a biological indicator of cool sea surface temperature, with special reference to New Zealand waters. J. R. Soc. N.Z. 20: 313-336

Henry, E. C. (1987). The life history of Phyllariopsis brevipes (= Phyllaria reniformis) (Phyllariaceae, Laminariales, Phaeophyta), a kelp with dioecious but sexually monomorphic gametophytes. Phycologia 26: 17-22

Hooper, R. G. (1984). Functional adaptations to the polar environment by the arctic kelp, Laminaria solidungula. Br. phycol J. 19: 194

Kain, J. M. (1964). Aspects of the biology of Laminaria hyperborea. III. Survival and growth of gametophytes. J. mar biol. Ass. U.K. 44: 415-433

Kain, J. M. (1969). Aspects of the biology of Laminaria hyperborea. V. Comparisons with early stages of competitors. J. mar. biol. Ass. U.K. 49: 455-473

Lewis, R. J. (1991). Intergeneric hybridization and chromosome numbers in kelps of the family Lessoniaceae. J. Phycol. 27 (Suppl.): 45

Lüning, K. (1980). Critical levels of light and temperature regulating the gametogenesis of three Laminaria species (Phaeophyceae). J. Phycol. 16: 1-15
Lüning, K. (1984). Temperature tolerance and biogeography of seaweeds: the marine algal flora of Helgoland (North Sea) as an example. Helgoländer Meeresunters. 38: 305-317

Lüning, K. (1990). Seaweeds. Their environment, biogeography and ecophysiology. John Wiley \& Sons, New York

Lüning, K., Freshwater, W. (1988). Temperature tolerance of northeast Pacific marine algae. J. Phycol. 24: 310-315

Lüning, K., Gurry, M. D., Masuda, M. (1987). Upper temperature tolerance of North Atlantic and North Pacific geographical isolates of Chondrus crispus (Rhodophyta). Helgoländer Meeresunters. 41:297-306

Lüning, K., tom Dieck, I. (1990). The distribution and evolution of the Laminariales: North Pacific-Atlantic relationships. In: Garbary, D. J., South, G. R. (eds.) Evolutionary biogeography of the marine algae of the North Atlantic. NATO A.SI Series, Vol. G 22. Springer-Verlag, Berlin, p. 187-204

Maier, I. (1984). Sexualität bei Braunalgen aus der Ordnung Laminariales und die Phylogenie der Ordnung. Ph.D. thesis, Univ. Konstanz

Mathieson, A. C., Norton, T. A., Neushul, M. (1981). The taxonomic implications of genetic and environmentally induced variations in seaweed morphology. Bot. Rev. 47 : 313-347

Munda, I. M., Lüning, K. (1977). Growth performance of Alaria esculenta off Helgoland. Helgoländer wiss. Meeresunters. 29: 311-314

North, W. J. (1972). Observations on populations of Macrocystis. In: Abott, I. A., Kurogi, M. (eds.) Contributions to the systematics of benthic marine algae of the North Pacific. Jap. Society of Phycology, Kobe, p. 75-92

Norton, T. A. (1977). Experiments on the factors influencing the geographical distributions of Saccorhiza polyschides and Saccorhiza dermatodea. New Phytol. 78: 625-635

Novaczek, I. (1984). Response of gametophytes of Ecklonia radiata (Laminariales) to temperature in saturating light. Mar. Biol. 82: 241-245

Novaczek, I., Bird, C. J., McLachlan, J. (1986). The effect of. temperature on development and reproduction in Chorda filum and C. tomentosa (Phaeophyta, Laminariales) from Nova Scotia. Can. J. Bot. 64: 2414-2420

Novaczek, I., Breeman, A. M. (1990). Thermal ecotypes of amphi-Atlantic algae. II. Cold-temperate species (Furcellaria lumbricalis and Polyides rotundus). Helgoländer Meeresunters. 44: 475-485

Novaczek, I., Lubbers, G. W., Breeman, A. M. (1990). Thermal ecotypes of amphi-Atlantic algae. I. Algae of Arctic to cold-temperate distribution (Chaetomorpha melagonium, Devalerea ramentacea and Phycodrys rubens). Helgoländer Meeresunters. 44: 459-474

Orfanidis, S. (1991). Temperature responses and distribution of macroalgae belonging to the warm-temperate Mediterranean-Atlantic distribution group. Botanica mar. 34: 541-552

Peters, A. F., Breeman, A. M. (1992). Temperature responses of disjunct temperate brown algae indicate long-distance dispersal of microthalli across the tropics. J. Phycol. 28: $428-438$

Peters, A. F., Breeman, A. M. (1993). Temperature tolerance and latitudinal range of brown algae from temperate pacific South America. Mar. Biol. 115: 143-150

Provasoli, L. (1968). Media and prospects for the cultivation of marine algae. In: Watanabe, A., Hattori, A. (eds.) Cultures and collections of algae. Proceedings of the U.S.-Japan conference, Hakone 1966. Japanese Society of Plant Physiology, Tokyo, p. 63-75

Saito, Y (1972). On the effects of environmental factors on morphological characteristics of Undaria pinnatifida and 
the breeding of hybrids in the genus Undaria. In: Abott, I. A., Kurogi, M. (eds.) Contributions to the systematics of benthic marine algae of the North Pacific. Japanese Society of Phycology, Kobe, p. 117-133

Saito, Y (1975). Practical significance of algae in Japan Undaria. In: Tokida, J., Hirose, H. (eds.) Advance of phycology in Japan. VEB Gustav Fischer Verlag, Jena, p. $304-320$

Sanbonsuga, Y., Neushul, M. (1978). Hybridization of Macrocystis (Phaeophyta) with other float-bearing kelps. J. Phycol. 14: $214-224$

Saunders, G. W., Druehl, L. D. (1991). Restriction enzyme mapping of the nuclear ribosomal cistron in selected Laminariales (Phaeophyta): a phylogenetic assessment. Can. J. Bot. 69: 2647-2654

Saunders, G. W., Druehl, L. D. (1992). Nucleotide sequence of the small-subunit ribosomal RNA genes from selected Laminariales (Phaeophyta): implications for kelp evolution. J. Phycol. 28: 544-549

Stam, W. T., Bot, P. V. M., Boele-Bos, S. A., van Rooij, J. M., van den Hoek, V. (1988). Single-copy DNA-DNA hybridizations among five species of Laminaria (Phaeophyceae): phylogenetic and biogeographic implications. Helgoländer Meeresunters. 42: 251-267

Sundene, O. (1962). The implications of transplant and culture experiments on the growth and distribution of Alaria esculenta. Nytt. Mag. Bot. 9: 155-174

Tatewaki, M. (1966). Formation of a crustose sporophyte with unilocular sporangia in Scytosiphon lomentaria. Phycologia 6: 62-66

tom Dieck, I. (1992). North Pacific and North Atlantic digitate Laminaria species (Phaeophyta): hybridization experiments and temperature responses. Phycologia 31: 147-163

tom Dieck, I., de Oliveira, E. C. (1993). South Atlantic and North Atlantic digitate Laminaria species (Phaeophyta):

This article was submitted to the editor hybridization studies and temperature responses. Mar Biol. 115: 151-160

van den Hoek, C. (1982). The distribution of benthic marine algae in relation to the temperature regulation of their life histories. Biol. J. Linn. Soc. 18: 81-144

van den Hoek, C., Breeman, A. M. (1990). Seaweed biogeography of the North Atlantic: Where are we now? In: Garbary, D. J., South, G. R. (eds.) Evolutionary biogeography of the marine algae of the North Atlantic NATO ASI Series, Vol. G22. Springer-Verlag, Berlin, p. $55-86$

Wiencke, C., tom Dieck, I. (1989). Temperature requirements for growth and temperature tolerance of macroalgae endemic to the Antarctic region. Mar. Ecol. Prog. Ser. 54: $189-197$

Wiencke, C., tom Dieck, I. (1990). Temperature requirements for growth and survival of macroalgae from Antarctica and southern Chile. Mar. Ecol. Prog. Ser. 59: $157-170$

Womersley, H. B. S. (1967). A critical survey of the marine algae of southern Australia. 2. Phaeophyta. Aust. J. Bot. 15: $189-270$

Yarish, C., Breeman, A. M., van den Hoek, C. (1984). Temperature, light and photoperiod responses of some Northeast American and West European endemic rhodophytes in relation to their geographic distribution. Helgoländer Meeresunters. 38: 273-304

Yarish, C., Breeman, A. M., van den Hoek, C. (1986). Survival strategies and temperature responses of seaweeds belonging to different biogeographic distribution groups Botanica mar. 29: 215-230

Yarish, C., Kirkman, H., Lüning, K. (1987). Lethal exposure times and preconditioning to upper temperature limits of some temperate North Atlantic red algae. Helgoländer Meeresunters. 41: 323-327

Manuscript first received: January 19, 1993

Revised version accepted: June 29, 1993 